\title{
Big Data und Wissen über Gesellschaft - die Quantifizierung des Sozialen
}

Auch wenn der erwartete Nutzen im Rahmen der Bedeutung des Wissens bereits durchgeklungen ist, muss die Verbindung der zentralen Elemente der vorliegenden Untersuchung, des Erkenntnis- und Nutzengewinns, vor dem Hintergrund der öffentlichen Wahrnehmung digitaler Datenverwertung erläutert und veranschaulicht werden. So ist an dieser Stelle zunächst die Bedeutung von Wissen mit Fokus auf Big Data vor aktuellen gesellschaftlichen Entwicklungen und hiermit verbundenen Nutzenerwartungen zu erörtern. Diese Nutzenerwartungen sollen hierbei mit Blick auf datenverarbeitende Wissensanwendungen anhand zweier Ebenen verdeutlicht werden: der Nutzen für das Individuum und der Nutzen für die Gesellschaft.

Dabei muss nun auch der Datenbegriff noch einmal aufgegriffen und präzisiert werden. Es ist nicht irgendeine Funktion der Daten und es sind auch nicht irgendwelche digitalen Daten, die hier mit Blick auf das Soziale relevant sind, sondern ihr Funktionsbezug der Bewertung und Verwertung. Digitale Daten ganz allgemein und nur für sich betrachtet sind zunächst nicht zwingend numerische Wertzuweisungen. Auch Texte, Bilder oder audiovisuelle Medien fallen zunächst unter den Datenbegriff, da sie mit Hilfe numerischer Werte in einer binär codierten Sequenz von Zustandsbeschreibungen von den IuK gespeichert und verarbeitet werden. Wenn allerdings im Zusammenhang mit Big Data als sozialem Phänomen von einer Datafizierung, Metrifizierung oder auch Quantifizierung der Gesellschaft oder des Sozialen gesprochen wird (Filipović, 2015; Holtzhausen, 2016; Mau, 2017; Merry, 2016; Passoth \& Wehner, 2013; M. T. Schäfer \& van Es, 2017) ${ }^{1}$, dann betrifft dies die Überführung realweltlich zu beobachtender Phänomene materieller oder immaterieller Natur in digitale Daten

\footnotetext{
${ }^{1}$ In der vorliegenden Arbeit wird dem Begriff der Quantifizierung (des Sozialen) Vorzug gegeben, da unter Metrifizierung auch die Einführung metrischer Einheitssysteme verstanden
} 
mit dem vorrangigen Ziel, diese so digitalisierten Entitäten zählbar voneinander unterscheidbar und somit vergleichbar sowie bewertbar zu machen (Hubbard, 2010). Der Zusammenhang der Bewertung und hiermit verbundener Vergleichung stellt dann neue Informationen über die Welt bereit, die Eingang in Wissenssysteme finden können. Auf diesem Wege kann nicht nur der Wissensbegriff mit Leben gefüllt werden, sondern gleichzeitig unter dem Vorzeichen einer gesamtgesellschaftlichen Bedeutung von Wissen auch dessen Nutzen diskutiert werden.

Quantifizierung meint hierbei zunächst eine Übersetzungsleistung oder wie Mau (2017) verdeutlicht: „Phänomene, Eigenschaften oder Beschaffenheiten eines Sachverhalts werden in einer allgemeinen, abstrakten und universell anschlussfähigen Sprache repräsentiert, der der Mathematik“ (S. 27). Daten entstehen hier vor allem auch durch das Messen, also einer proaktiven ${ }^{2}$ Erhebung von Daten, bei der entsprechend ein empirisches Relativ in ein numerisches Relativ überführt wird (Rössler, 2017; Schnell et al., 2018). Hierbei wird im Rahmen der Messung ein vorher festgelegtes Regelwerk herangezogen, so dass Messen konkret auch wie folgt verstanden wird (Stevens, 1946): "The assignment of numerals to objects or events according to rules" (S. 677). Allerdings geht es zumindest im Rahmen sozialwissenschaftlicher Fragestellungen genauer um die Eigenschaften der beobachteten Objekte oder Ereignisse, denen Zahlenwerte zugewiesen werden (Bandalos, 2017; Hand, 2016). Dabei trägt wie in Kapitel 3 dokumentiert die Verbreitung von IuK und ihre stetige Präsenz in der sozialen Situation einen gewichtigen Teil zu dieser Quantifizierung bei.

Doch die bloße Möglichkeit der Übersetzung in das digitale Datenformat sagt noch nichts über dessen tatsächliche Notwendigkeit aus, Entitäten zu digitalisieren und in diesem Moment mit Zahlenwerten zu belegen. Selbst wenn, wie in Kapitel 4 dargelegt, Wissen als ein Wert an sich betrachtet wird und die Wissensgesellschaft sich die Produktion, Organisation und Verwertung von Wissen zur Aufgabe gemacht hat, ist diese Erklärung zunächst noch nicht hinreichend.

wird und Datafizierung vor allem auf den technischen Übersetzungsprozess von Entitäten in Daten abstellt.

${ }^{2}$ Hierzu ist anzumerken, dass die Übersetzungsleistung zwar aktiv angeregt muss, also den IuK per Command-Zeile befohlen wird. Allerdings ist hiermit jedoch noch nicht verbunden, dass sich auch bewusst für die Messung entschieden wurde. Die Registrierung von digitalen Repräsentationen der Subjekte und Objekte sowie von Ereignissen, Orten und Zeiten geschieht laufend und beiläufig, weshalb der Begriff der Messung hier zunächst weiter zu fassen ist, als in der standardisierten empirischen Sozialforschung (zu diesem Messbegriff siehe Abschnitt 8.1). Die Messung im vorliegenden Sinne ist also nicht zwingend systematisch und zielgerichtet, doch auf jeden Fall nicht-zufällig. 
Wie angemerkt, muss insbesondere der Nutzen in den Blick genommen werden, der aus diesem Wissen gezogen wird. Es ist daher notwendig, auf die besondere soziale Qualität und hierbei die spezifische gesellschaftliche Affordanz des Wissens abzustellen, um zu verdeutlichen, warum und wie individuelle und gesellschaftliche Nutzengewinne imaginiert und idealisiert werden. Erst die sich ergebenden Möglichkeiten der Unterscheidung, der Bewertung und eines anschließenden Vergleichs weisen auf den Nutzengewinn durch die weitreichende Quantifizierbarkeit sozialer Phänomene hin, die sich aus den vielfältigen Möglichkeiten der Generierung von Daten im Rahmen der eingangs besprochenen Digitalisierung ergeben. Dieses Verhältnis von sozialer Quantifizierung und Nutzengewinn vor dem Hintergrund gesellschaftlicher Vergleichslogik bedarf daher näherer Betrachtung. Hierbei ist die zuvor aufgemachte Perspektive individueller und gesellschaftlicher Nutzenerwartungen eng miteinander verwoben und folgt nun zunächst einem übergeordneten Argument, in dessen Rahmen sich beide nicht immer voneinander trennen lassen.

\subsection{Zur Bedeutung von Zahlen im sozialen Kontext}

Zunächst ist hier auf die generelle soziale Bedeutung von Zahlen abzustellen. Der Wissenschaftshistoriker Cohen zeigt in seiner geschichtlichen Abhandlung The Triumph of Numbers (2006), wie Zählen und Messbarmachen sich aus eurozentrischer Perspektive im Zeitverlauf entwickeln und zu Beginn vor allem zur nationalstaatlichen und wissenschaftlichen Entwicklung beigetragen haben. Die (Sozial-)Statistik sorgte dafür, die allgemeine Anwendbarkeit und den Nutzen von Zahlen und mathematischen Bezügen zu demonstrieren und zum Durchbruch auf breiter gesellschaftlicher Ebene zu verhelfen. Dabei wurde bereits vielfach die Entwicklung und Bedeutung der Verwendung von Zahlen und Statistiken für die Herausbildung des modernen Nationalstaats nach westfälischer Staatenordnung hervorgehoben (Crook \& O'Hara, 2011; Desrosiéres, 1998; Hacking, 1990; MacKenzie, 1981; T. M. Porter, 2011; Rottenburg et al., 2015). „Das quantitative Wissen über Territorium und Bevölkerung stellt seit jeher eine wesentliche Voraussetzung staatlicher Handlungskapazität [sic], denn erst wenn Dinge benannt und statistisch erfasst worden sind, können sie zum Gegenstand politischer Interventionen werden“ (Mau, 2017, S. 186-187). Mit Hilfe der Auszählung bspw. von männlichen Rekruten und ihrer physischen Verfasstheit ergab sich die Möglichkeit der präzisen militärischen Organisation und Planung. Es konnte abgeschätzt werden, wie viele Soldaten im Kriegsfall zur Verfügung standen, worauf sich Ausrüstung und Logistik einzustellen hatten, wie umfangreich die Finanzierung 
(Stichwort: Steuern) auszusehen hatte und es war ein Vergleich mit den Heeren anderer Länder möglich (Cohen, 2006). Die Vorteile, die sich aus dem Wissen dieser Daten ergaben, gingen einher mit der Einrichtung staatlicher Institutionen zur kontinuierlichen Datenerhebung und -auswertung. Es entstand ein weitreichender Verwaltungsapparat, da man nunmehr regionale Gebiete als eine Einheit zusammenfassen musste, um einen geografischen Raum und die in ihm lebenden Merkmalsträger erfassen zu können. Die Vorzüge der quantitativen Erfassung von Phänomenen im Rahmen von Bestandsaufnahme und Planung waren also eng verknüpft mit (politischer) Handlungsmacht und führten dazu, dass diese fortan auf weitere Gesellschaftsbereiche ausgedehnt wurde.

\section{Qualitative Wertzuweisungen innerhalb der quantitativen Logik}

Dabei erlauben Zahlen nicht nur eine quantitative Beschreibung im Sinne des Auszählens, sondern können nach der Logik der Messung und der Unterscheidung zwischen den Messniveaus auch qualitative Eigenschaften betreffen und dokumentieren. Die Qualität von etwas wird im Rahmen einer Valenzzuordnung positiv oder negativ beschrieben, so dass eine qualitative Bewertung auch als „evaluatives Einsortieren in Dualismen, Rangfolgen und Skalen“ (Reckwitz, 2017, S. 66, Hervor. im Orig.) verstanden werden kann, anhand dessen Wertzuweisungen unterschieden und zueinander in Beziehung gesetzt werden (Vormbusch, 2012).

Bewertungen auf Basis quantitativer Wertzuweisungslogik eröffnen durch die vorgeblich saubere mathematische Ordnung der Umwelt einen rationalen und übersichtlichen Zugang zu dieser (Mau, 2017) und gehen einher mit einem weitgefassten Bestreben nach formaler taxonomischer Ordnung sowie Standardisierung (Higgins \& Larner, 2010; Timmermans \& Epstein, 2010). So durchzieht das kontinuierliche Abzählen und Wertzuweisen durch Quantifizierung mittlerweile alle gesellschaftlichen Bereiche (Lamont, 2012; Power, 1997): Wie in Kapitel 3 deutlich geworden ist, sind es mithin bei weitem nicht nur staatliche Institutionen, die im Rahmen einer staatlichen Steuerungslogik für alles Mögliche Daten erheben, oder im Rahmen einer ökonomischen Verwertungslogik agierende Unternehmen, die Produktion, Absatz und Konsum von Produkten und Dienstleistungen verdaten und optimieren. Auch auf der Ebene des Individuums wird das Selbst im Rahmen des Self-Trackings der laufenden Datenerhebung unterworfen (siehe Abschnitt 3.3). Den vermeintlichen Nutzen gesellschaftlicher Quantifizierung in den Blick nehmend, wird mithin auf den funktionalen Charakter digital quantifizierter Wertzuschreibungen abgestellt werden, der Unterscheidungen des Disparaten ermöglicht (Nassehi, 2019). Hier sind unlängst etliche soziologische Erklärungsansätze entstanden, die allesamt auch auf die durch digitale Daten 
bedingte Unterscheidungs- und Vergleichslogik abstellen und die vorliegende empirische Analyse der Erkenntnis- und Nutzenerwartungen bezüglich digitaler Daten argumentativ vorbereiten (Heintz, 2010, 2016; Mau, 2017; Nassehi, 2019; Reckwitz, 2017).

\subsection{Zahlen als Grundlage von Bewertungen und Vergleichen}

Heintz (2010, 2016) folgend begründen sich Phänomene wie Metrifizierung, Quantifizierung, Datafizierung auf einer Soziologie des Vergleichs. Werden Entitäten mit Zahlen belegt und bewertet, können eigentlich inkommensurable Dinge durch Überführung in ein numerisches Format vergleichbar und wie zuvor ausgeführt leichter kategorisiert sowie hierarchisiert und somit in eine Wert- und Präferenzordnung überführt werden. Zum einen werden also Entitäten durch die Zuordnung von Zahlen und dadurch expliziten Wertzuweisungen zunächst als prinzipiell vergleichbar angesehen und zum anderen dann dennoch durch die bezifferte Wertigkeit unterschieden. Oder wie Heintz (2016) ausführt:

Vergleiche beruhen also auf zwei Operationen, die empirisch zwar zusammenlaufen, analytisch aber zu unterscheiden sind: auf der Einstufung der Vergleichsobjekte als vergleichbar (Kategorisierung) und auf der Feststellung ihrer (graduellen) Differenz anhand eines tertium comparationis. Es ist diese Kombination von Gleichheitsunterstellung und Differenzbeobachtung, die die Besonderheit von Vergleichen ausmacht. (S. 307, Hervorh. im Orig.)

So wird der Vergleich, den die Sozialwissenschaften als methodischen Ansatz der Empirie kanonisiert haben, zur sozialen Praxis (Heintz, 2016). Denn dieser Vergleich ermöglicht und fördert zum einen insbesondere eine Statusbestimmung innerhalb der nunmehr sichtbar gemachten Strukturen und dient somit der vereinfachten Feststellung sowie Selbstvergewisserung und hierauf aufbauender Optimierung von (Eigen-)Wert. Zunächst ist diese Praxis allgemein vor dem Hintergrund der Rationalität von Präferenzordnungen eines sich selbst als Homo Oeconomicus beschreibenden nutzen-maximierenden Menschen zu bewerten (Kirchgässner, 2013). Aus der Vergleiche ermöglichenden Sozialkalkulation kann in der kapitalistischen Moderne ein Profit generiert werden, etwa wenn die unterschiedliche Leistung und Leistungsfähigkeit von Arbeitskräften von den Human Resources zueinander in Bezug gesetzt werden (Vormbusch, 2012). Insbesondere mit Blick auf das Subjekt und seine Identität findet diese dann 
jedoch auch im Rahmen eines Impression Managements im Rahmen von OnlineKommunikation Anwendung, wenn Selbstbild und -wert anhand der Anzahl virtueller Freunde und sogenannter Likes festgemacht werden. ${ }^{3}$

Reckwitz (2017) unterscheidet mit Blick auf die von ihm analysierte Gesellschaft der Singularitäten, in der die Unterscheidungen des Allgemeinen und Besonderen zentrale Kategorien darstellen, in vier Sorten von Praktiken. Bezüglich der angesprochenen Vergleichslogik ist auch hier insbesondere das Bewerten und weiterhin das Hervorbringen von Wertordnungen kalkulativer Praxis im Rahmen der Quantifizierung des Sozialen von Interesse. Dieses ist jedoch eng verknüpft mit dem vorhergehenden Beobachten und dem nachfolgenden Aneignen. Hierzu merkt Reckwitz (2017) an: „Die vier genannten Sorten von Praktiken sind nur heuristisch zu trennen, denn es ist möglich, dass sie eng miteinander verzahnt sind oder sogar miteinander kombiniert auftreten (so dass etwa in der gleichen Praxis hergestellt und rezipiert wird). Sie können auch hochgradig spezialisiert und ausdifferenziert nebeneinanderstehen und sich zu ganzen institutionellen Komplexen verdichten“ (S. 64, Hervorh. im Orig.). Als ein solcher institutioneller Komplex ist die Quantifizierung des Sozialen mit Hilfe von Digitaltechnik zu verstehen.

Auch wenn der Ansatz von Statuseinschätzung und Selbstvergewisserung sowie anschließender Verwertung womöglich nicht zu einer umfassenden und abschließenden Erklärung taugt, da diese selbst etwa unter den Vorzeichen übergeordneter kapitalistischer oder anderweitiger idiosynkratischer Logiken erklärungswürdig sind: ${ }^{4}$ Die Konsequenz ist, dass ein jeder, der einen irgendwie gearteten Nutzen aus der Mustererkennung und der eigenen Positionsverortung mittels vergleichender Bewertung ziehen möchte, sich selbstredend selbst der Logik der umfassenden und standardisierten Evaluation unterwerfen muss und somit das Fortschreiten der gesellschaftlichen Prozesse dieser Valorisierung begünstigt (Lamont, 2012; Muniesa, 2012; Reckwitz, 2017). Wie Mau (2017) diesbezüglich resümiert: „Permanente Vermessung und Bewertung führen dazu, dass sich sowohl die Fremd- als auch die Selbststeuerungsleistungen intensivieren" (S. 13).

${ }^{3}$ Zum allg. Zusammenhang von Status und Identität allgemein siehe Abels (2017), insb. Abschnitt 22.3. Mit Fokus auf virtuelle Online-Welten siehe Chester und Di Bretherton (2009), Fullwood (2019) sowie Laurent und Bouzefrane (2015).

${ }^{4}$ Die spezifische funktionale Logik von Wissen um Bewertung und Vergleich ist immer situativ im jeweiligen Verwertungszusammenhang zu verstehen. Siehe z. B. die Bedeutung von Vergleichshorizonten und Nutzungsmotiven im Zusammenhang mit Gamification (Sailer, 2016). 
Eine einmal festgestellte (Un-)Gleichheit informiert dann mithin Anschlusshandlungen als Reaktion auf die Differenzbeobachtungen, die aus der jeweiligen inhärenten situativen Vergleichslogik folgen. Beispielhaft zeigt sich dann im Spitzensport und der Wirtschaft, wer gerade zur Leistungselite zählt, in Kunst und Kultur kann festgestellt werden, welche Kunstschaffenden, Stile oder Werke gerade en vogue sind, und auch in der von der Wählergunst abhängigen Politik zeigt sich, welche politischen Programme und Personen aktuell reüssieren. Diesbezügliche Erkenntnisse lassen sich auch und insbesondere unter den Vorzeichen der Ökonomie recht wechselhafter Aufmerksamkeit (Franck, 1998; Schroer, 2014) entsprechend verwerten. So erhält der Hollywoodstar mit den meisten Likes den lukrativen Werbevertrag. Oder man ist vielleicht auch einfach nur eher geneigt ein Buch zu lesen, das bei eben jenen Leuten populär ist, die auch den eigenen Lieblingsroman positiv bewertet haben. In Analogie zu den zuvor beschriebenen Algorithmen werden die festgestellten Unterscheidungen und Bewertungen folglich im Rahmen von Entscheidungsprogrammen zur Lösung bereichsspezifischer Probleme herangezogen. Es kann dann vom Modus einer Zustandsfeststellung in den Modus des zielgerichteten Handelns mit dem Ziel der Zustandsveränderung gewechselt werden.

\section{3 luK als Motor globaler Vergleichshorizonte}

Zunächst ist jedoch näher auf die IuK als Motor globaler Vergleichshorizonte einzugehen. IuK und die durch sie produzierten digitalen Daten eignen sich hierbei aufgrund ihrer immanenten mathematischen Übersetzungsleistung besonders auch für einen zu automatisierenden fortlaufenden Einsatz im Funktionszusammenhang der gesellschaftlichen Quantifizierung. Sie wirken somit vereinfachend auf die umfangreiche Fortentwicklung und Ausbreitung gesellschaftlicher Quantifizierung des Sozialen, da sie in besonderem Maße den an diesen Vergleichszusammenhang gestellten Anforderungen genügen:

Das Entstehen eines überlokalen, universalistischen Vergleichszusammenhangs setzt 1) die kontinuierliche Produktion öffentlicher Vergleichsereignisse voraus, denn nur dann kann kontinuierlich verglichen und laufend zwischen unterschiedlichen Vergleichsresultaten differenziert werden; sie setzt 2) die Herstellung von Vergleichbarkeit dieser Vergleichsereignisse jenseits ihrer lokalen Entstehungskontexte voraus, denn nur was unter bestimmten Gesichtspunkten plausibel als gleich beschrieben werden kann, kann unter anderen Gesichtspunkten plausibel als ungleich beschrieben werden; schließlich setzt es 3) die Etablierung von Vergleichskriterien voraus, die die 
Ereignisse in einen übergreifenden Vergleichszusammenhang integrieren. Gleichzeitig wird damit die Möglichkeit geschaffen, Ereignisse auf einer Zeitachse einzuordnen und sie mit anderen, vergangenen und zukünftigen Vergleichen in Beziehung zu setzen. (Heintz \& Werron, 2011, S. 365)

So zeigt sich auch diesbezüglich die vermeintliche Leistungsfähigkeit digitaler Technik und hiermit einhergehender Entstehung und Verwertung digitaler Daten. Anstatt, dass Unterscheidungen und hierauf aufbauende Vergleiche laufend durch den Menschen getroffen und verarbeitet werden müssen, wird die kalkulative Praxis an die IuK ausgelagert und durch datenverarbeitende Algorithmen in standardisierter Form festgeschrieben, was eine gleichbleibende Qualität der Vergleiche in Datenform, ihrer Verarbeitung und der produzierten Ergebnisse verspricht. Mit der Konsequenz, dass dieses ,Erfolgsmodell‘ selbstredend auch Vergleiche jenseits nationalstaatlicher Grenzen zulässt oder vielmehr erfordert.

Die Logik von Wertzuweisungs- und Vergleichsprozessen gepaart mit den Nutzungsmöglichkeiten der IuK hat eine grenzüberschreitende Anziehungskraft und die Durchsetzung des Vergleichs ist diesbezüglich auch als Voraussetzung und Treiber von Globalisierungstendenzen zu analysieren und kann zum Verständnis von global übergreifenden Entwicklungen in unterschiedlichen gesellschaftlichen Feldern herangezogen werden (Bühler \& Heintz, 2017; Heintz, 2012; Heintz \& Werron, 2011). Wie bereits aufgezeigt, ist der Preis für Einsichten der verheiBungsvollen Selbstverortung für alle Teilnehmenden gleich und besteht in einer Unterwerfung unter das Regelwerk von Standardisierung und Quantifizierung, das individuelle Eigentümlichkeiten ignoriert (Mau, 2017). So demonstrieren Heintz und Werron (2011) die Möglichkeit von Globalisierung durch die Entstehung globaler Vergleichshorizonte insbesondere am Beispiel der Wissenschaft und des Spitzensports. Doch gibt es auch in vielen anderen gesellschaftlichen Teilbereichen mittlerweile global institutionalisierte Vergleichslogiken (Blank, 2007; Erdi, 2019; Espeland \& Sauder, 2007; Ringel \& Werron, 2019; Sauder \& Espeland, 2009).

Damit Vergleiche hier ihre globalgesellschaftliche Wirkmächtigkeit entfalten können, müssen sie zunächst weitläufig kommuniziert werden. Der Vergleich darf also nicht nur auf der mentalen Ebene verbleiben, sondern muss in einer sozialen Situation durch Kommunikation Eingang finden (Heintz, 2016). Denn nur, wenn Wissen um Differenz auf Grundlage eines Vergleichs dem breiten öffentlichen Diskurs zugeführt wird, entfaltet er gesellschaftliche Folgen. So blieben Hochschulrankings folgenlos, wenn nur wenige oder gar niemand von ihnen lesen oder erfahren würde, wenn sie also keinen Eingang in die mediale Berichterstattung oder die externe Kommunikation der Hochschulen selbst fänden (Heintz \& 
Werron, 2011). Erst wenn sie der öffentlichen Debatte zugeführt werden, können sie Bedeutung erhalten und führen dazu, dass Studierende, Forschende und andere Beteiligte sich in ihrem Handeln an ihnen orientieren. Dies drückt sich dann bspw. in der Entscheidung aus, sich aufgrund eines festgestellten Unterschieds in der Qualität von Forschung und Lehre an Hochschule A zu bewerben und nicht an Hochschule B.

Vor allem Entwicklungen auf der technologischen und organisationalen Ebene - und hier insbesondere die internationale Ausweitung massenmedialer Kommunikation - begünstigten die Ausdehnung von Vergleichshorizonten. Ein illustratives Beispiel für die Verbreitung der Vergleichslogik im Sport liefern Heintz und Werron (2011) selbst:

Die ,epochale ' Leistung der Telegraphie bestand dabei darin, ein Kommunikationsnetz bereitzustellen, mit dessen Hilfe sich Beobachtungs- und Beschreibungsknoten wie Sportverbände oder Presseredaktionen einrichteten, wo Wettkampfinformationen zusammengeführt, notiert, gesammelt, verglichen, evaluiert und, für unser Argument entscheidend, publiziert wurden mit der Folge, dass nunmehr die gleichzeitige Informiertheit aller Ausübenden und Interessierten einer Sportart unterstellt werden konnte. (...) Die Allianz aus Presse und Telegraphie war insofern stilbildend für eine auch heute noch gültige Kombination aus elektrischen Kommunikationstechnologien und statistisch-narrativer öffentlicher Evaluation, die von weiteren Medien und Formen der Berichterstattung im 20. Jahrhundert nur noch ausgeweitet und verfeinert werden konnte. (S. 378-379)

Die vorhergehenden Ausführungen verdeutlichen, dass sich der inhärente Nutzen dieser gesellschaftlichen Verständigung über Quantifizierung aus den aus ihr entspringenden Möglichkeiten einer globalen Bewertungs- und Vergleichslogik ergibt. Diese erlaubt die Verortung von Subjekten und Objekten sowie räumlicher und zeitlicher Ereignisse innerhalb individueller oder bereichsspezifischer Werteund Präferenzordnungen. Es lässt sich daher leicht erahnen, welchen Schub die Möglichkeiten vernetzter IuK in den globalen Vergleichszusammenhängen derzeit entfalten. Die kalkulative Vergleichslogik diffundiert dabei nicht nur global in alle Sphären öffentlichen und privaten Lebens, sondern findet in diesem Rahmen wie gezeigt auch Eingang in gesellschaftliche Kommunikationsprozesse. Im Zuge der globalen Digitalisierung wird die Möglichkeit umfassender Ausdehnung der Bewertungs- und Vergleichshorizonte durch die Quantifizierung des Sozialen als Grundlage von Steuerungs- und Optimierungsprozeduren hier nun auch zum Anspruch umformuliert und verkehrt sich so womöglich zu einer kollektiven Erwartungshaltung. 


\subsection{Die Wahrnehmung großer digitaler Datenbestände vor dem Hintergrund der Quantifizierung des Sozialen}

Im Rahmen der Ausdehnung der Quantifizierung des Sozialen und der Entstehung und Verwertung entsprechender digitaler Datenbestände wird in der Literatur vielfach diskutiert, inwieweit die zuvor skizzierten Entwicklungen nun auch menschliche Vorstellungen von Realität und sozialer Wirklichkeit formen. In einem nun nachfolgend zu beschreibenden Wahrnehmungsmuster von Big Data fügen sich die in den vorangegangenen Kapiteln angesprochenen Ausführungen zusammen und es wird herausgearbeitet wie eine entsprechende Wahrnehmung aussehen und welche Folgen für eine Erwartungshaltung bezüglich der Quantifizierung sie haben könnte.

\section{Wahrnehmung und Konsequenzen kalkulativer Praktiken}

Vormbusch (2012) postuliert zunächst ganz allgemein zur Konsequenz kalkulativer Praxis: „Welche Vorstellungen gesellschaftlicher Wirklichkeit realitätsmächtig werden, ist auch durch den historisch spezifischen Gebrauch organisierter Zahlensysteme bestimmt. Mit diesen werden jeweils bestimmte Handlungsmöglichkeiten und -restriktionen etabliert, entlang derer soziale Akteure ihre Strategien und Möglichkeiten ausloten“ (S. 20). Es wird im Rahmen kalkulativer Praktiken wie institutionalisierter Messverfahren und hierauf fußender Wertordnungen wie Rankings in der Folge soziales Anpassungsverhalten beobachtet: Espeland und Sauder (2007) sprechen diesbezüglich auch von Reaktivität, Mackenzie (2006) von Performativität und Hacking (2007) von dynamischem Nominalismus. ${ }^{5}$

Mau (2017) überträgt diese Reaktionen und ihre Konsequenzen anschaulich auf die Folgen der Quantifizierung des Sozialen im Rahmen von Big Data:

Mit der Verfügung über immer mehr Daten begibt sich die Gesellschaft auf den Weg zu einer datengetriebenen Prüf-, Kontroll- und Bewertungsgesellschaft, die nur noch das glaubt, was in Zahlen vorliegt. Soziale Selbsterkenntnis und Regulierung beziehen sich inzwischen so intensiv auf Daten, dass das Erkennen dessen, was ist, ohne sie kaum mehr möglich erscheint. (S. 46)

\footnotetext{
${ }^{5}$ In der Konsequenz auf diese Beobachtungen formuliert Campbell (1979) folgendes Gesetz: "The more any quantitative social indicator is used for social decision-making, the more subject it will be to corruption pressures and the more apt it will be to distort and corrupt the social processes it is intended to monitor" (S. 85).
} 
Eine durch Big Data geprägte Weltsicht kann dahingehend charakterisiert werden, als dass in der durch IuK angetriebenen kalkulativen sozialen Praxis der Quantifizierung des Sozialen überaus großes Potential für Erkenntnis- und Nutzengewinne gesehen wird. Diese Weltsicht ist also durch eben jene Potentiale von Big Data geprägt, die in Abschnitt 3.5 im Rahmen der sozio-technischen Betrachtung digitaler Datenbestände als konsequentiell-evaluative Dimensionen von Big Data besprochen wurden. Die zuvor thematisierten Versprechungen und Idealvorstellungen der Qualitäten von digitalen Daten werden dabei kommunikativ weiterverbreitet und weitgehend von der Bevölkerung übernommen. Ein Mythos lebt mithin davon, dass man ihn sich selbst und anderen immer wieder erzählt und seine wesentlichen Aussagen dabei beständig und affirmierend wiederholt werden (Barthes, 1964).

Der Mythos Big Data bezieht sich in seiner originären Beschreibung bei boyd und Crawford (2012) zunächst lediglich auf die epistemische Qualität der Daten und ihre Konsequenz für die Möglichkeiten von Wissen.

Big data, it is implied, is the source of a different order of knowledge, a step change in human self-understanding that precisely bypasses humans' meagre attempts at selfunderstanding through interpreting the local details of what they think, say and do. (Couldry, 2017, S. 235)

Die besondere Qualität von Big Data baut also auf der in Abschnitt 4.2 angesprochenen Bedeutung von Wissen und dessen funktionalem Potential für prozedurale Steuerung auf. Gefragt wurde hier jedoch zunächst nach den Möglichkeiten digitaler Daten für Wissen, nicht nach den Erwartungen, die der Mythos womöglich evoziert. Diesbezüglich thematisiert Degele (2000) im Zusammenhang mit der Computerisierung womöglich

tiefgreifende Folgen (...) im stillen Wandel der Konstituierung von Wissen. (...) Im Zuge dessen werde sich eine neue Wissensordnung ausbilden, in welcher sich ein spezifischer, datenerzeugender Denkstil mit überwiegend flachem (Rohstoff-)Wissen in Wissenschaft und Gesellschaft ausbreiten werde. Das reine Datenwissen gewinne damit eine erstrangige Bedeutung und lege die Ausbildung einer spezifischen Rationalitätsform für daten- statt theoriezentriertes Problemlösen nahe. (S. 14)

Insbesondere mit Blick auf Self-Tracking diskutieren Sharon und Zandbergen (2016) den Vorwurf eines innerhalb der Quantified-Self-Bewegung vorherrschenden Daten-Fetischismus, der von ihnen wie folgt charakterisiert wird: "The widespread idea that what draws self-trackers to numerical data is its perceived power of truth and objectivity" (S. 1695). Die besondere epistemische Qualität 
von digitalen Daten und deren Bedeutung für Wissen sind im Umkehrschluss auch von wesentlicher Bedeutung für die Wahrnehmung von Big Data: Es kann am besten auf Grundlage digitaler Daten gewusst werden, so dass alles, was und worüber gewusst werden soll, in digitale Daten überführt werden muss.

Mit dem Verwertungs- und Steuerungspotential von Daten verbunden ist also weiterhin die Erwartung an einen Nutzen, welcher sich, wie gezeigt, auf unterschiedlichen Ebenen zwischen Individuum und Kollektiv manifestieren kann. Dabei ist der konkrete Nutzen zwar bereichsspezifisch jeweils völlig anders geartet, in seiner Valenz jedoch durchweg positiv. In Fragen der Mobilität ist ein Nutzen aus Datensammlung und -auswertung also anders gelagert als in der Medizin oder der Wirtschaft, jedoch ist er immer als Gewinn verstanden. Die positive Färbung ist, wie gezeigt, zum einen bedingt durch das Entstehen und die Förderung digitaler Datentechnik und IuK im wissenschaftlich-industriellen Komplex, welcher ein besonderes Interesse an der Hervorhebung ihrer Vorteile hat. Zum anderen wird sie jedoch auch allen Mitgliedern der Gesellschaft zugeschrieben. Diesen attestiert Schirrmacher (2009) einen „Wahn, aus Angst vor Kontrollverlust die Welt in Formeln, Systematiken und Algorithmen, kurzum in Mathematik zu verwandeln. Wir werden immer unfähiger, mit Unsicherheiten und Unwahrscheinlichkeiten umzugehen (...)“ (S. 32). Als Ergebnis führen der Umgang mit dem Computer und die Auslagerung des Denkens und Entscheidens an die Digitaltechnik dazu, dass sich der menschliche Denkapparat verwandelt (Schirrmacher, 2009). Die gewichtige Konsequenz, die sich aus der Kombination wahrgenommener Erkenntnis- und Nutzenpotentiale der Daten in der Folge ergibt: „Wir erleben gerade in Echtzeit, wie eine Gesellschaft unwiderruflich die Fundamente ihres Weltbildes ändert" (Schirrmacher, 2009, S. 221).

Für Nassehi (2019) hingegen verändert die fortschreitende Digitalisierung nicht eben jenes gesellschaftliche Fundament, sondern ist ein elementarer Teil dessen: Sie ist für ihn ,nicht nur eine soziale Erscheinung (...), sondern sogar ein soziologisches Projekt" (S. 18, Hervorh. im Orig.). So wie im Rahmen der Quantifizierung des Sozialen der empirische Ansatz des Messens zur sozialen Praxis wird, ist ,,vieles von dem, was die Digitalisierung betreibt, (...) von geradezu soziologischer Denkungsart: Sie nutzt soziale Strukturen, sie macht soziale Dynamiken sichtbar und sie erzeugt aus diesen Formen der Mustererkennung ihren Mehrwert“ (S. 18). Digitalisierung sei ,unmittelbar verwandt (...) mit der gesellschaftlichen Struktur" (S. 18, Hervorh. im Orig.). Die Möglichkeiten, Muster und Strukturen mit Hilfe von Digitaltechnik sichtbar zu machen, fallen eben daher auf so fruchtbaren Boden, da sie bereits auf eine entsprechende Erwartungsstruktur der Gesellschaft treffen - diese ist mithin nicht Konsequenz, sondern Bedingung von Digitalisierung. 
Ganz gleich, welche Vorbedingungen, Ursachen oder konkreten Änderungen des Denkens und Beobachtens in Zusammenhang mit Big Data diagnostiziert werden: In der Literatur wird eine aus der Wahrnehmung der Digitalisierung abgeleitete Erwartungshaltung immer schon unter dem Vorzeichen ihrer bereits manifesten, meist negativen Konsequenzen für Individuum und Gesellschaft diskutiert. Es wird mithin nicht nur vor den Risiken und Gefahren selbst gewarnt, die mit dem unhinterfragten Einsatz der Systeme einhergehen (Eubanks, 2018; Zuboff, 2019) und sich aus den negativen Konsequenzen der Quantifizierung für Individuum und Gesellschaft ergeben (Muller, 2019; O'Neil, 2017; Tufekci, 2015). Es wird auch konkret vor einer kollektiven Mentalität gewarnt, die sich aus der stetigen Wiederholung der Ansprüche an datenbasiertes Wissen ergibt und die eine kritische Analyse und Reflexion der gesellschaftlichen Diffusion von Techniken der Quantifizierung verhindert oder zumindest erschwert (Mau, 2017; Merry, 2016; Schirrmacher, 2009). Für einen Daten-Fetischismus gilt dann bspw.: "As it seeks to reduce all phenomena and means of accounting for phenomena to numbers, it simultaneously displaces other less easily quantifiable albeit insightful ways of expressing phenomena" (Sharon \& Zandbergen, 2016, S. 1698, Hervorh. im Orig.).

Kalkulative Praktiken im Rahmen einer technokratischen Steuerungslogik von Gesellschaft

Die Wahrnehmung bezüglich der Potentiale von IuK für Wissen, Verstehen, Denken und hierauf begründeten Nutzen ist dort, wo sie angesprochen wird, also immer schon präsent. Diese Diagnose der Konsequenz kalkulativer Praktiken schließt dabei an eine lange Tradition von Beschreibungen und Warnungen bezüglich vermeintlich weit verbreiteter irrationaler Wahrnehmungsmuster technokratischer gesellschaftlicher Steuerungslogik an, die sich bspw. bereits in den Arbeiten von Arendt (1972) und Roszak (1995) finden lassen. So kritisiert Arendt (1972) die Denkmuster der Entscheidungstragenden innerhalb des Pentagons im Zuge des Vietnamkrieges: "The problem-solvers did not judge; they calculated. (...) An utterly irrational confidence in the calculability of reality, becomes the leitmotif of the decision-making processes (...)" (S. 37-39, Hervorh. im Orig.).

Roszak (1995) skizziert diese spezifische Logik einer von ihm analysierten technokratischen Gesellschaft demnach wie folgt:

[A] society in which those who govern justify themselves by appeal to technical experts who, in turn, justify themselves by appeal to scientific forms of knowledge. And beyond the authority of science, there is no appeal. (...) Its assumptions about reality and its values become as unobtrusively pervasive as the air we breathe. (S. 7-8) 
Auch hier steht der vermeintliche evidenzgetriebene Erkenntnisgewinn, das, was als scientific form of knowledge beschrieben wird, im Mittelpunkt gesellschaftlicher Wahrnehmungsmuster und prägt eine Erwartungshaltung bezüglich der Entscheidungspraktiken innerhalb der eingangs beschriebenen Wissensgesellschaft. Diese findet nun unter den Vorzeichen von Computertechnik und digitaler Daten verstärkt Widerhall.

Weizenbaum (1976) thematisiert dieses technokratische Weltbild entsprechend früh mit Blick auf die aufkommende Computerisierung: "The introduction of computers into our already highly technological society has [...] merely reinforced and amplified those antecedent pressures that have driven man to an ever more highly rationalistic view of his society and an ever more mechanistic image of himself" (S. 11). Er beschreibt dabei sein Erstaunen über die fehlende menschliche Einsicht zu reflektieren, zu welchem Denken eine Maschine den Menschen befähigt, der die Maschine zum Denken befähigt (vgl. auch Turkle, 1984). Die Konsequenz, den ein maschinenzentrierter Blick auf Vorstellungen der Denkprozesse von Mensch und Computer im Vergleich zu einem menschenzentrierten Blick hat, wird in der Folge von Norman (1993) herausgearbeitet: Während der menschenzentrierte Blick den Menschen bspw. als flexibel und veränderungsbereit versteht und den Computer als starr, spricht der maschinenzentrierte Blick dem Computer die Fähigkeit logischen und organisierten Denkens zu und dem Menschen ebenjene ab.

Es muss festgestellt werden, dass die kritische Reflexion der Beziehung von Individuum und Gesellschaft zu Digitaltechnik und allen voran Computern mit Blick auf Vorstellungen und Denken über die Welt keineswegs neu ist. Dieser Kritik an einer technokratisch geprägten Wahrnehmung von Gesellschaft und Technik, der (Fehl-)Wahrnehmung der Potentiale und Differenzen menschlichen und computerisierten Denkens und in der Fortsetzung auch an der durch IuK getriebenen Quantifizierung des Sozialen ist jedoch gemein, dass sie bereits immer auf das Vorhandensein dieser Wahrnehmungsmuster und hieraus resultierender Erwartungshaltungen im Sinne einer kollektiven Prädisposition abstellt, die je nach Betrachtung mit der Digitalisierung oder ihrer jeweiligen Artefakte und Phänomene verbunden ist.

Auch die verheißungsvolle Erzählung der ubiquitären Verdatung mit ihren Möglichkeiten für computerisierte Formen des Denkens und Verstehens von Welt hat hier bereits immer schon gesellschaftliche Resonanz gefunden und mündet darauffolgend in entsprechend beeinflussten Einstellungen und Handlungen. Sie wird als Ergebnis eines kontinuierlichen und konsonanten gesellschaftlichen Diskurses vorausgesetzt, in dem Wirtschaft, Wissenschaft, Politik und Medien als dominante Sprecher auftreten. So resümiert Mau (2017): 
Dass Daten zur Leitwährung des gesellschaftlichen Selbstbeobachtungs- und Selbstbeschreibungsgeschäfts geworden sind, ist allzu offensichtlich. Inwieweit sie andere Beschreibungs- und Beurteilungsformen des Sozialen nicht nur marginalisieren, sondern vollständig zu verdrängen vermögen, ist bislang eine offene Frage. Mit der Durchsetzung der Quantifizierung werden wir aber - mehr oder weniger - zu Gläubigen in der Kirche der Zahlen. (S. 47)

Diese deterministisch anmutende Sichtweise, bei der eine eingangs skizzierte Wahrnehmung der Quantifizierung des Sozialen zu einer entsprechenden Erwartungshaltung führt, greift meiner Ansicht nach zu kurz. Erst muss der Versuch unternommen werden, etwas über das Vorhandensein entsprechender Annahmen und Erwartungen - oder das, was Mau auch als einen Glauben bezeichnet zu erfahren. Es muss also zunächst die tatsächliche Struktur und Ausprägung der Überzeugungen bezüglich der gewonnenen Erkenntnis und des Nutzens aus der Quantifizierung analysiert werden, bevor das Vorhandensein einer kollektiven Mentalität faktisch angenommen und etwaige Auswirkungen dieser untersucht werden können.

Open Access Dieses Kapitel wird unter der Creative Commons Namensnennung 4.0 International Lizenz (http://creativecommons.org/licenses/by/4.0/deed.de) veröffentlicht, welche die Nutzung, Vervielfältigung, Bearbeitung, Verbreitung und Wiedergabe in jeglichem Medium und Format erlaubt, sofern Sie den/die ursprünglichen Autor(en) und die Quelle ordnungsgemäß nennen, einen Link zur Creative Commons Lizenz beifügen und angeben, ob Änderungen vorgenommen wurden.

Die in diesem Kapitel enthaltenen Bilder und sonstiges Drittmaterial unterliegen ebenfalls der genannten Creative Commons Lizenz, sofern sich aus der Abbildungslegende nichts anderes ergibt. Sofern das betreffende Material nicht unter der genannten Creative Commons Lizenz steht und die betreffende Handlung nicht nach gesetzlichen Vorschriften erlaubt ist, ist für die oben aufgeführten Weiterverwendungen des Materials die Einwilligung des jeweiligen Rechteinhabers einzuholen.

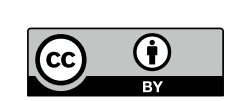

